\title{
Dominance of Interface Chemistry over the Bulk Properties in Determining the Electronic Structure of Epitaxial Metal/Perovskite Oxide Heterojunctions
}

ARTICLE in CHEMISTRY OF MATERIALS · MAY 2015

Impact Factor: $8.35 \cdot$ DOI: 10.1021/acs.chemmater.5b01118

READS

61

6 AUTHORS, INCLUDING:

\section{Scott A Chambers}

Pacific Northwest National Laboratory

320 PUBLICATIONS 9,929 CITATIONS

SEE PROFILE

Timothy Droubay

Pacific Northwest National Laboratory

128 PUBLICATIONS 3,177 CITATIONS

SEE PROFILE

\section{Meng Gu}

University of California, Davis

70 PUBLICATIONS $\mathbf{1 , 4 0 6}$ CITATIONS

SEE PROFILE

Peter V Sushko

University College London

131 PUBLICATIONS 3,073 CITATIONS

SEE PROFILE 


\title{
Dominance of Interface Chemistry over the Bulk Properties in Determining the Electronic Structure of Epitaxial Metal/Perovskite Oxide Heterojunctions
}

\author{
Scott A. Chambers, ${ }^{* \dagger}{ }^{\dagger}$ Yingge Du, ${ }^{\ddagger}$ Meng Gu, ${ }^{\ddagger}$ Timothy C. Droubay, ${ }^{\dagger}$ Steven P. Hepplestone, ${ }^{\S}$ \\ and Peter V. Sushko ${ }^{\dagger}$ \\ ${ }^{\dagger}$ Fundamental and Computational Sciences Directorate, Physical Sciences Division and ${ }^{\ddagger}$ Environmental Molecular Sciences \\ Laboratory, Pacific Northwest National Laboratory, Richland, 99352 Washington United States \\ ${ }^{\S}$ Department of Physics and Astronomy, University College London, Gower Street, London, WC1E 6BT, United Kingdom
}

\begin{abstract}
We show that despite very similar crystallographic properties and work function values in bulk $\mathrm{Fe}$ and $\mathrm{Cr}$, epitaxial films of these metals on $\mathrm{Nb}: \mathrm{SrTiO}_{3}(001)$ exhibit completely different heterojunction electronic properties. The $\mathrm{Cr} / \mathrm{SrTiO}_{3}$ interface is ohmic, whereas $\mathrm{Fe} / \mathrm{SrTiO}_{3}$ forms a Schottky barrier with a barrier height of $0.50 \mathrm{eV}$. This difference arises because of variations in interface chemistry. In contrast to $\mathrm{Cr}$ [Chambers, S. A. et al., Adv. Mater. 2013, 25, 4001.], in-diffused Fe exhibits a +2 oxidation state and occupies $\mathrm{Ti}$ sites in the perovskite lattice, resulting in negligible charge transfer to $\mathrm{Ti}$, upward band bending, and Schottky barrier formation. The differences between $\mathrm{Cr}$ and $\mathrm{Fe}$ are understood by performing first-principles calculations of the energetics of defect formation, which corroborate experimental results.
\end{abstract}

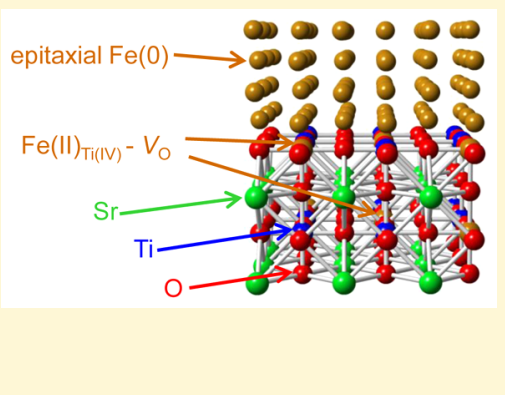

\section{INTRODUCTION}

Ohmic contact formation on wide-gap semiconductors using a single metal is a formidable challenge. The primary limitation is that the band edges in materials with large bandgaps typically do not align well with metal Fermi edges, precluding facile carrier transport across the interface. This problem is inherent to all wide-gap materials, including fluorides, nitrides, and oxides. In considering wide-gap oxides such as $\mathrm{SrTiO}_{3}$ (STO), the vast majority of pure metals form Schottky barriers. ${ }^{1-3}$ The standard approach to mitigating this problem is to deposit one or more reactive metals, usually taken from $\mathrm{In}, \mathrm{Cr}, \mathrm{Ni}$, $\mathrm{Ti}$, and $\mathrm{Al}^{4}{ }^{4}$ often followed by deposition of a $\mathrm{Au}$ capping layer. ${ }^{5}$ Although an ohmic contact generally results, this approach has the disadvantage of process complexity due to the need to deposit multiple metals and the appearance of a polycrystalline interface, which is undesirable from a uniformity point of view, especially for nanoscale device fabrication. A better approach is to find pure, single metal ohmic contacts, preferably epitaxial, for which the structural and electronic uniformity are likely to be high. Cr has been found to be such a metal. It grows epitaxially on $\mathrm{STO}(001)$, despite a $+4.3 \%$ in-plane lattice mismatch, and forms a nearly perfect ohmic contact with a contact resistivity of $29 \mathrm{~m} \Omega \cdot \mathrm{cm} .{ }^{6}$ The reason for this unexpected, but very useful behavior is that during initial nucleation, some $\mathrm{Cr}$ atoms diffuse into the STO and occupy interstitial sites within the first few atomic planes. ${ }^{7}$ This diffusion process results in two highly desirable features. First, in-diffused $\mathrm{Cr}$ atoms anchor the rest of the film, resulting in the expected epitaxial relationship, $(001)_{\mathrm{Cr}} \|(001)_{\mathrm{STO}}$ and $[100]_{\mathrm{Cr}}$ \| $[110]_{\text {STO }}$. Second, interstitial Cr transfers charge to nearby $\mathrm{Ti}$ ions at $\mathrm{B}$ sites in the perovskite lattie and causes the bands to bend down, thereby metalizing the surface and creating what is, in effect, a metal/metal interface with the expected ohmic behavior.

It is of interest to see if other pure metals exhibit the same properties as $\mathrm{Cr}$ on STO. To this end, we performed a computational study of several transition metals as interstitial dopants in the vicinity of the STO(001) surface. Using the density functional theory (DFT) and a standard generalized gradient approximation functional, ${ }^{8}$ we investigated the electronic properties of interstitial metal atoms and characterized the local lattice distortions and the amount of charge donated by each interstitial species in STO. These calculations revealed that $\mathrm{Fe}$ has a higher binding energy than $\mathrm{Cr}$ at the interstitial sites near the surface of STO and donates only slightly less charge than $\mathrm{Cr}$ to the neighboring Ti cations. Bulk Fe metal exhibits a bcc structure, like $\mathrm{Cr}$, and has a lattice parameter of $2.86 \AA$, which is very similar to that of $\mathrm{Cr}(2.88$ $\AA$ ). Thus, $\mathrm{Fe}$ should, and has indeed been shown to grow epitaxially on STO with very similar structural properties as Cr. ${ }^{9,10}$ Additionally, polycrystalline $\mathrm{Cr}$ and $\mathrm{Fe}$ have very similar work functions $(\sim 4.5 \mathrm{eV} \text { for } \mathrm{Cr}, \sim 4.7 \mathrm{eV} \text { for } \mathrm{Fe})^{11}$ and so these two metals are expected to give rise to similar Schottky barrier heights based on simple models. Thus, it is of considerable interest to directly compare the electronic properties and interface structures for $\mathrm{Fe} / n-\mathrm{STO}(001)$ and $\mathrm{Cr} / n-\mathrm{STO}(001)$ heterojunctions prepared under the same conditions.

Received: March 25, 2015

Revised: May 13, 2015 


\section{METHODS}

Experiments. Fe and $\mathrm{Cr}$ epitaxial films were deposited from separate effusion cells on $0.5 \mathrm{wt} \% \mathrm{Nb}$-doped STO(001) substrates by means of molecular beam epitaxy (MBE) at a substrate temperature of $550{ }^{\circ} \mathrm{C}$. The substrates were prepared by etching in high-purity DI water, followed by tube furnace annealing in air for $8 \mathrm{~h}$ at $1000{ }^{\circ} \mathrm{C}$. This treatment results in a predominantly $\mathrm{TiO}_{2}$ terminated surface, which is readily cleaned in the MBE chamber by heating at $500{ }^{\circ} \mathrm{C}$ in $5 \times$ $10^{-6}$ Torr $\mathrm{O}_{2}$ for $20 \mathrm{~min}$. The Fe film thicknesses range from 0.25 monolayer (ML, defined as the $\mathrm{Ti}$ atom density on STO(001), $\left.6.54 \times 10^{14} \mathrm{~cm}^{-2}\right)$ up to $700 \mathrm{ML}(100 \mathrm{~nm})$. The $100 \mathrm{~nm}$ films were deposited through a shadow mask with a Van der Pauw hole pattern consisting of $1 \mathrm{~mm}$ diameter holes at the corners of a square with $l=w=5 \mathrm{~mm}$ for transport measurements. Thick films of $\mathrm{Fe}$ were also grown on $\mathrm{MgO}(001)$ under the same conditions, but without a shadow mask, to determine the effect of surface oxidation on the measured electronic properties. All films except those grown with the shadow mask were characterized in situ by means of reflection high-energy electron diffraction (RHEED) and X-ray and ultraviolet photoemission spectroscopy (XPS and UPS) using a VG/Scienta R3000 electron energy analyzer and monochromatized AlK $\alpha$ X-ray and He I $\alpha$ UV light. The XPS and UPS energy resolutions were $0.50 \mathrm{eV}$ and $10 \mathrm{meV}$, respectively. Ex situ characterization included atomic force microscopy (AFM), scanning transmission electron microscopy and electron energy loss spectroscopy (STEM/EELS) using a $300 \mathrm{kV}$ FEI Titan microscope, and transport measurements using a Quantum Design Physical Properties Measurement System or Ecopia HMS-5000. Specimens were prepared for STEM/EELS by a wedge polishing method using a Multiprep apparatus from Allied High Tech Products, Inc. The STEM convergence angle was approximately $18 \mathrm{mrads}$, and the collection semiangle for the Z-contrast imaging was in the range of 55-270 mrads. The Gatan image filter (GIF) used in this analysis was a Quantum 965 with dual EELS capability, which enables simultaneous acquisition of the zero-loss peak and high-loss regions. The GIF CCD camera was a $2000 \times$ 2000 pixel model, which allowed us to simultaneously acquire the zero-loss peak and the $\mathrm{O} K, \mathrm{Ti} L$, and $\mathrm{Cr} L$ edges. The zeroloss peak was used to calibrate the energy loss scale. The EELS collection semiangle was $\sim 93$ mrads.

Modeling. The STO bulk was represented using the periodic model and a supercell comprised of $2 \times 2 \times 5$ cubic perovskite unit cells. The same supercell was used to model the STO(001) surface; in this case, a vacuum gap of $15 \AA$ was used to separate the periodically repeated STO slabs. The calculations were performed using the Vienna ab initio simulation package (VASP). ${ }^{12,13}$ The projected augmented wave (PAW) method was used to approximate the electronion potential. ${ }^{14}$ Exchange-correlation effects were treated within the Perdew-Burke-Ernzerhoff (PBE) functional form of the GGA, modified for solids (PBEsol). ${ }^{15}$ The plane-wave basis with a $500 \mathrm{eV}$ cutoff was used. The calculations were performed using the $3 \times 3 \times 2$ (bulk) and $3 \times 3 \times 1$ (surface) Monkhorst-Pack k-point mesh with its origin at the $\Gamma$ point. The charge and spin density distributions were analyzed using the Bader method. ${ }^{16}$ The lattice cell parameters were fixed at the bulk STO values corresponding to $a_{0}=3.905 \AA$. The energies of self-consistent calculations were converged to $10^{-6}$ $\mathrm{eV} /$ cell, and atomic positions were relaxed until the forces on the ions were less than $0.02 \mathrm{eV} / \AA$. The GGA+U approach to describe the on-site electronic correlations of the $\mathrm{Ti}, \mathrm{Cr}$, and $\mathrm{Fe}$ $3 \mathrm{~d}$ orbitals, where the parameters $U$ were selected so as to reproduce the band gap of STO, $\mathrm{Cr}_{2} \mathrm{O}_{3}$, and $\mathrm{Fe}_{2} \mathrm{O}_{3}$, respectively $\left(U_{\mathrm{Ti}}=8 \mathrm{eV}, U_{\mathrm{Cr}}=3 \mathrm{eV}, U_{\mathrm{Fe}}=5 \mathrm{eV}\right){ }^{17}$

\section{RESULTS AND DISCUSSION}

Similar to $\mathrm{Cr}$, Fe nucleates epitaxially on $n$-STO(001). However, the growth mode for Fe is Volmer-Weber (3D islands) to a greater extent than for Cr. Figure 1 shows a summary of structural and morphological data establishing these results.
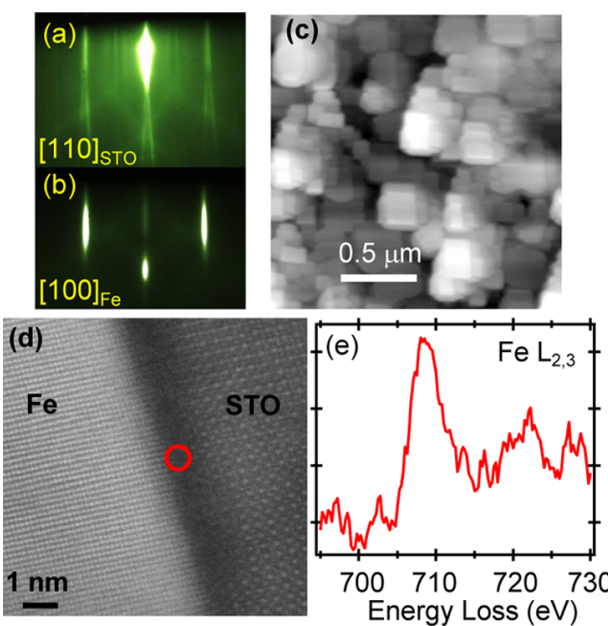

Figure 1. Structural and morphological data for $54 \mathrm{~nm}$ epitaxial Fe on $n$-SrTiO ${ }_{3}(001)$ : RHEED patterns for the clean substrate (a) and after Fe deposition (b) with the incident beam aligned along the [110] direction in the substrate, AFM image (c, $2 \mu \mathrm{m} \times 2 \mu \mathrm{m} \times 30 \mathrm{~nm})$, STEM image (d), and Fe L-edge EELS (e) measured inside the circle shown in (d).

The RHEED patterns (Figure 1a and $b$ ) reveal that epitaxial growth occurs according to the expected epitaxial relationships $(001)_{\mathrm{Fe}} \|(001)_{\mathrm{STO}}$ and $[100]_{\mathrm{Fe}} \|[110]_{\mathrm{STO}}$. The spacing between diffracted beams in the film RHEED pattern with the beam incident along $\langle 100\rangle$ is given by $d_{100}^{*} \propto 1 / a_{\mathrm{Fe}}$ and that for the substrate with the incident beam along $\langle 110\rangle$ is given by $d_{110}^{*} \propto \sqrt{2} / a_{\mathrm{STO}}$, where $a_{\mathrm{Fe}}$ and $a_{\mathrm{STO}}$ are the in-plane lattice parameters. Because $a_{\mathrm{STO}} \cong \sqrt{ } 2 a_{\mathrm{Fe}}$, it follows that $d_{100}^{* \mathrm{Fe}} \cong d_{110}^{* \mathrm{STO}}$ if indeed $(001)_{\mathrm{Fe}} \|(001)_{\mathrm{STO}}$ and $\langle 100\rangle_{\mathrm{Fe}} \|\langle 110\rangle_{\mathrm{STO}}$. Inspection of the RHEED patterns in Figure 1a and $b$ shows that the streak spacings for the Fe film are very close to those for the STO substrate when the beam is oriented along $\langle 110\rangle_{\text {STO }}$. The STO:Fe streak spacing ratio in this azimuth $\left(d_{110}^{* \mathrm{STO}} / d_{100}^{* \mathrm{Fe}}\right)$ equals 1.03 which is quite close to the value expected if the Fe film is fully relaxed, 1.04. The 3D epitaxial island growth pattern is clearly seen in the AFM image in Figure 1c. The presence of rather wide $(\sim 100 \mathrm{~nm})$ islands with flat terraces gives rise to the coherent surface diffraction seen in the film surface RHEED pattern (Figure 1b). RHEED patterns for thinner films are dominated by transmission through these islands when the island dimensions are less than the attenuation length for the $15 \mathrm{keV}$ incident RHEED beam. A representative STEM image for the interface in Figure $1 \mathrm{~d}$ also reveals the same structural relationship established by the RHEED data, identical to that for $\mathrm{Cr} / \mathrm{STO}(001)$. 
The epitaxial relationship is where the similarity with $\mathrm{Cr}$ / $\mathrm{STO}(001)$ ends. The $\mathrm{Fe} / \mathrm{STO}(001)$ interface includes a disordered region (Figure 1d) which contains $\mathrm{Fe}$, according to the EELS spectrum (Figure 1e) measured within the circle in Figure 1d. Additionally, Fe forms a Schottky barrier. The $I-V$ curves measured at room temperature are shown in Figure 2a.

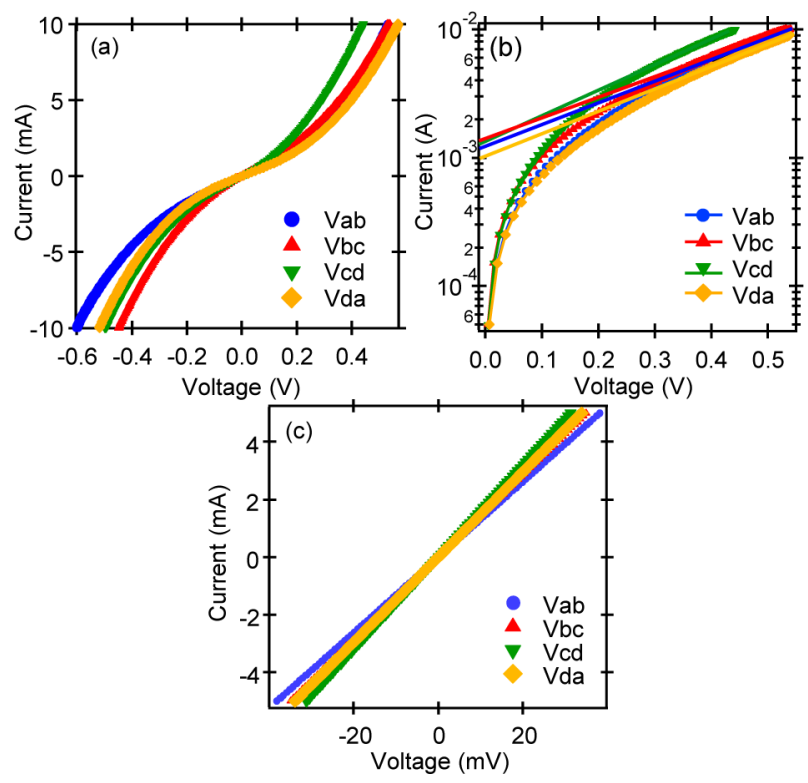

Figure 2. Room-temperature $I-V$ curves for $100 \mathrm{~nm} \mathrm{Fe}$ on $n$ $\mathrm{SrTiO}_{3}(001)$ (a and b), and for a blanket $100 \mathrm{~nm}$ epitaxial $\mathrm{Fe}$ film on $\mathrm{MgO}(001)(c)$.

Rectifying behavior is seen for all four configurations of applied current and measured voltage, although there is some difference in the detailed behavior. The ideality factor is $\sim 1.2$. Figure $2 \mathrm{~b}$ shows the extrapolated saturation currents at zero voltage for the four configurations. These vary from $1.0 \mathrm{~mA}$ to $1.4 \mathrm{~mA}$. The Schottky barrier height is given by $\varphi_{B}=(k T / q)$ $\ln \left(A A^{*} T^{2} / I_{\mathrm{s}}\right)$, where $A$ is the diode area, $A^{*}$ is the Richardson constant which is $120\left(\mathrm{~m}^{*} / \mathrm{m}_{0}\right), \mathrm{m}^{*}$ being the effective mass for free carriers in STO, and $I_{s}$ is the saturation current at zero bias. The range of $I_{s}$ determined from Figure 2b, yields a barrier height of $0.50-0.51 \mathrm{eV}$. Due to the high reactivity of Fe with oxygen, it is conceivable that the measured Schottky barrier is due, at least in part, to a rectifying contact which forms at the $\mathrm{Fe}$ oxide/Fe metal interface resulting from air exposure. To investigate this possibility, a control experiment was carried out in which a blanket $100 \mathrm{~nm}$ epitaxial Fe film was grown on insulating $\mathrm{MgO}(001)$ at the same substrate temperature used for $\mathrm{Fe} / \mathrm{STO}$ heterojunction formation. XPS shows that air exposure of this film results in the formation of a few $\mathrm{nm}$ of $\mathrm{Fe}_{2} \mathrm{O}_{3}$. The resulting $I-V$ curves, which directly probe the $\mathrm{Fe}_{2} \mathrm{O}_{3} / \mathrm{Fe}$ interface (Figure 2c), were perfectly linear, establishing that surface oxidation of the Fe film does not introduce anomalies into the $\mathrm{Fe} / \mathrm{STO}$ transport measurements.

The substantial differences in electronic properties for $\mathrm{Fe}$ and $\mathrm{Cr}$ on $n-\mathrm{STO}(001)$ are due to differences in the chemistry of interface formation. Unlike $\mathrm{Cr}$, which occupies interstitial sites, ${ }^{7}$ Fe occupies lattice sites. Furthermore, the $\mathrm{Cr} L_{2,3}$ edge EELS indicates that $\mathrm{Cr}$ in STO is predominantly $\mathrm{Cr}(\mathrm{III})$, while the $\mathrm{Fe}$ $L_{2,3}$ energy loss values and $L_{3}$ to $L_{2}$ intensity ratio seen in Figure 1e are characteristic of a +2 oxidation state. ${ }^{18}$ The nearinterface STO region imaged in Figure $1 \mathrm{~d}$ is not sufficiently well ordered to determine the location of Fe within the lattice. To gain a clearer picture, sub-ML quantities of $\mathrm{Fe}$ and $\mathrm{Cr}$ were deposited separately on clean $n$-STO $(001)$ substrates, at the same temperature $\left(550{ }^{\circ} \mathrm{C}\right)$ and deposition rate $(\sim 1 \mathrm{ML} / \mathrm{min})$ as used to deposit the thicker $\mathrm{Fe}$ and $\mathrm{Cr}$ films, followed by overgrowth of a $\sim 18 \mathrm{~nm}$ thick STO film at $650{ }^{\circ} \mathrm{C}$. The sub$\mathrm{ML} \mathrm{Fe}$ and $\mathrm{Cr}^{7}$ on $\mathrm{STO}(001)$ interface properties were investigated by in situ XPS and UPS prior to STO overgrowth, and the buried $\mathrm{STO} / \mathrm{Fe}, \mathrm{Cr} / \mathrm{STO}$ interfaces were interrogated using ex situ STEM/EELS.

As with in-diffused Fe resulting from thick-film growth, subML Fe exhibits primarily a +2 charge state, as seen in XPS Fe $2 p$ spectrum shown in Figure 3a. Also shown for reference are

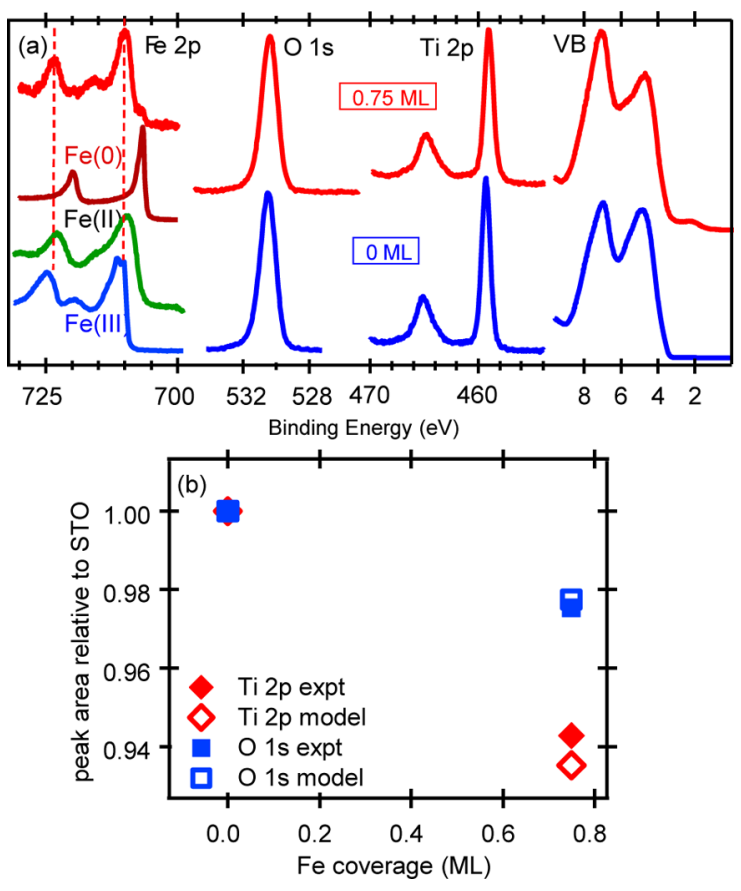

Figure 3. Core-level and valence band X-ray photoelectron spectra for $0.75 \mathrm{ML} \mathrm{Fe}$ on $n-\mathrm{SrTiO}_{3}(001)$ (a), along with Ti $2 \mathrm{p}$ and $\mathrm{O}$ 1s corelevel peak areas normalized to those for clean $n$ - $\mathrm{SrTiO}_{3}(001)$ (closed symbols) (b). Also shown in (b) are model calculations of the peak area ratios (open symbols), as described in the text. The standards used for the Fe $2 p$ reference spectra in (a) were epitaxial films of $\mathrm{Fe}$ on $\mathrm{STO}(001), \mathrm{FeO}$ on $\mathrm{MgO}(001)$, and $\alpha-\mathrm{Fe}_{2} \mathrm{O}_{3}$ on $\alpha-\mathrm{Al}_{2} \mathrm{O}_{3}(0001)$.

spectra for epitaxial films containing $\mathrm{Fe}$ in its different oxidation states. The binding energies of all principal features observed in the Fe $2 p$ spectra for coverages up to and including $0.75 \mathrm{ML} \mathrm{Fe}$ films match well those for $\mathrm{FeO}(001)$. However, starting at 0.75 $\mathrm{ML}$, a very small $\mathrm{Fe}(0)$ feature becomes visible, suggesting that $0.75 \mathrm{ML}$ represents a solubility limit for $\mathrm{Fe}$ in STO at $550{ }^{\circ} \mathrm{C}$.

A structural model for the sub-ML Fe/STO(001) interface can be deduced by considering the attenuation of the Ti $2 \mathrm{p}$ and $\mathrm{O}$ 1s peak areas upon $\mathrm{Fe}$ deposition, guided by theoretical calculations. As discussed below, there is no evidence from STEM that sub-ML Fe resides at interstitial sites in the STO. Therefore, we consider lattice site configurations for the $\mathrm{Fe}(\mathrm{II})$. Our DFT calculations reveal that $\mathrm{Fe}$ (II) is not stable on the A sites, where $\mathrm{Sr}$ normally resides. Indeed, the local atomic structure of the A site is such that the Fe $3 \mathrm{dt}_{2 g}$ orbitals for $\mathrm{Fe}_{\mathrm{Sr}}$ substitutional defects would be less stable than the $\mathrm{e}_{\mathrm{g}}$ orbitals due to their orientation relative to $\mathrm{O} 2 \mathrm{p}$ orbitals on the adjacent anions. As a result, the six $3 \mathrm{~d}$ electrons of $\mathrm{Fe}(\mathrm{II})$ should fully 
occupy the $e_{g}$ orbitals and partially occupy the $t_{2 g}$ orbitals inducing a considerable local lattice distortion. In contrast, $\mathrm{Fe}(\mathrm{II})$ is predicted to be stable at the B sites, where Ti resides, provided an $\mathrm{O}$ vacancy is generated on the nearest anion position. Furthermore, the calculations predict that formation of a new $\mathrm{BO}_{2}$ layer of composition $\mathrm{Fe}_{x} \mathrm{Ti}_{1-x} \mathrm{O}_{2-x}$ atop the terminal $\mathrm{TiO}_{2}$ substrate layer would be stable for $x \leq 0.65 \mathrm{ML}$ Several aspects of the photoemission measurements support the assignment of such a new $\mathrm{BO}_{2}$ layer. First, the $\mathrm{O} 1 \mathrm{~s}$ and $\mathrm{Ti}$ $2 p$ line shapes do not change upon Fe deposition (Figure 3a), consistent with the absence of any secondary surface oxide phases (such as pure $\mathrm{FeO}$ ), and there is no reduction of $\mathrm{Ti}(\mathrm{IV})$.

The spectra exhibit a slight $(\sim 0.15 \mathrm{eV})$ reduction in binding energy for both core levels upon deposition of $0.75 \mathrm{ML} \mathrm{Fe}$, corresponding to a very small change in band bending relative to the clean surface, which is within $0.1 \mathrm{eV}$ of being in a flatband condition. A simple density-of-states calculation establishes that at the doping level employed here, the Fermi level is $14 \mathrm{meV}$ below the conduction band minimum in the flat-band condition. Likewise, the various features in the VB spectrum shift to a slightly lower binding following $\mathrm{Fe}$ deposition and a Fe-derived $3 \mathrm{~d} t_{2 \mathrm{~g}}$ feature appears in the gap, but well below the Fermi level (Figure 3a). The binding energy of this feature is consistent with the presence of $\mathrm{Fe}(\mathrm{II})$. There is also a marked reduction in intensity for the deeper $\mathrm{VB}$ feature at $\sim 7 \mathrm{eV}$, which has significant $\mathrm{Ti} 3 \mathrm{~d}$ character, relative to the feature at $\sim 4.5 \mathrm{eV}$, which is largely $\mathrm{O} 2 \mathrm{p}$ derived. This result is consistent with $\mathrm{Fe}$ substitution for $\mathrm{Ti}$ in the surface layer. Accordingly, we have modeled the attenuation of the $\mathrm{Ti} 2 \mathrm{p}$ and $\mathrm{O} 1 \mathrm{~s}$ intensities assuming that a new layer of $\mathrm{Fe}_{x} \mathrm{Ti}_{1-x} \mathrm{O}_{2-x}$ nucleates atop the terminal $\mathrm{TiO}_{2}$ layer when $0.75 \mathrm{ML} \mathrm{Fe}$ is deposited.

The apparent discrepancy between the negligible band bending in the STO measured by XPS for sub-ML films and the $0.5 \mathrm{eV}$ Schottky barrier height measured by $I-V$ curves for thick $(100 \mathrm{~nm})$ films is most likely due to film morphology. Even Fe depositions of up to $15 \mathrm{ML}$ do not lead to a measured deviation from the flat-band condition of more than $\sim 0.1 \mathrm{eV}$. However, neither the full electronic structure of the metal nor the final atomic structure of the interface is established by ML coverages. Moreover, because Fe films grow as 3D epitaxial islands (see Figure 1c), the portions of the STO substrate readily detectable by XPS are those with ultrathin Fe overlayers. Therefore, it should come as no surprise that the band bending measured by XPS should be different from the barrier height measured by transport through very thick Fe films.

We deduce the $\mathrm{Fe}_{x} \mathrm{Ti}_{1-x} \mathrm{O}_{2-x}$ interface structure using a simple attenuation model to predict $\mathrm{Ti} 2 \mathrm{p}$ and $\mathrm{O} 1 \mathrm{~s}$ intensities at normal emission relative to those for clean $\mathrm{STO}(001)$ for different distributions of $0.75 \mathrm{ML} \mathrm{Fe}$ in the near-surface region. An attenuation length of $1.5 \mathrm{~nm}$ was used in these calculations for both photoelectrons. Several different structures with $\mathrm{Fe}$ distributed within the top few $\mathrm{BO}_{2}$ layers were considered. Agreement is best for both core levels for the structure $\mathrm{Fe}_{x} \mathrm{Ti}_{1-x} \mathrm{O}_{2-x}-\mathrm{TiO}_{2}-\mathrm{SrO}-\mathrm{TiO}_{2}-\mathrm{SrO}-\ldots$ where $x=0.65$. The predicted and measured extents of attenuation are shown in Figure $3 \mathrm{~b}$. The possibility of diffusion of a minority of the 0.75 ML Fe deeper into the STO was not included in the model and could explain the $0.1 \mathrm{ML}$ discrepancy between the attenuation model and the deposited amount. This result is in excellent agreement with the predictions of DFT calculations. Here, the total energy of a system consisting of an STO supercell with four $\mathrm{Ti}$ sites per $\mathrm{BO}_{2}$ layer was calculated after adding one, two, three, and four Fe atoms from the gas phase and extracting the same number of $\mathrm{O}$ atoms and $\mathrm{O}-\mathrm{Ti}-\mathrm{O}$ moieties from the STO cell to form a $\mathrm{Fe}_{x} \mathrm{Ti}_{1-x} \mathrm{O}_{2-x}$ layer on the surface. A linear fit of the five calculated total energies for supercells with one to four $\mathrm{Fe}$ atoms crosses the zero energy cost line at $x=\sim 0.65$, suggesting that the system is stable for $\mathrm{Fe}_{\leq 0.65} \mathrm{Ti}_{\geq 0.35} \mathrm{O}_{\leq 1.35}$, as seen in Figure 4 .

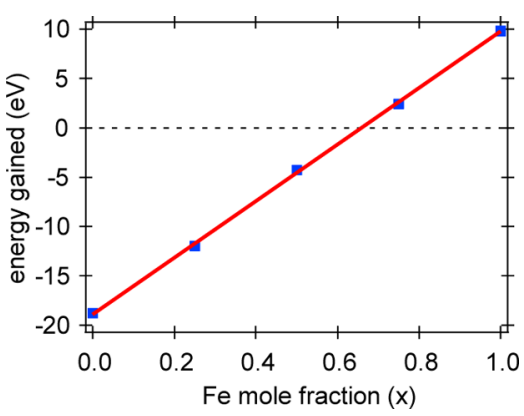

Figure 4. Total energy gain associated with adding Fe to $\mathrm{STO}(001)$ for $\mathrm{TiO}_{2}$-terminated $\mathrm{SrTiO}_{3}(001)$ with an additional layer of $\mathrm{Fe}_{x} \mathrm{Ti}_{1-x} \mathrm{O}_{2-x}$ layer as computed by density functional theory.

That $\mathrm{Fe}(\mathrm{II})_{\mathrm{Ti}(\mathrm{IV})}$ substitutional defects are still present when epitaxial STO is overgrown on $0.75 \mathrm{ML} \mathrm{Fe} / \mathrm{STO}$ interface is readily apparent from STEM HAADF images and $\mathrm{Fe}_{2,3}$ EELS spectra in Figure $5 a-c$. The dashed lines in the large-scale image (Figure 5a) show the location of the buried interfacial region. The high-resolution image (Figure $5 \mathrm{~b}$ ) reveals the structural coherence of the interface and does not show any intensity at interstitial sites. This result is in contrast to that observed in images for the analogous STO/Cr/STO(001)

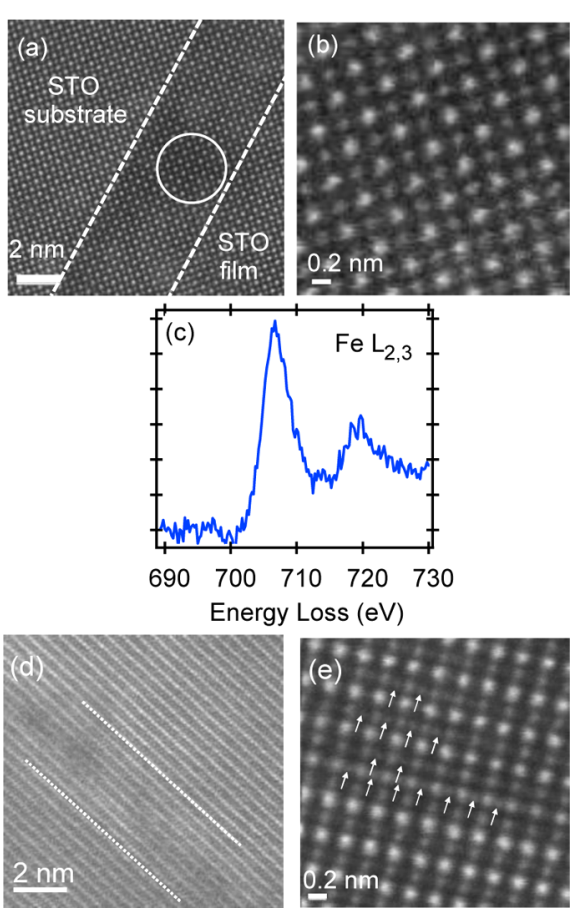

Figure 5. STEM images for $0.75 \mathrm{ML} \mathrm{Fe}$ on $n-\mathrm{SrTiO}_{3}(001)$ viewed along $\langle 001\rangle$ zone axis (a and b), along with Fe L-edge EELS (c) measured within the circle shown in (b). Also shown are STEM images for $0.90 \mathrm{ML} \mathrm{Cr}$ on $n$-SrTiO $3(001)$ viewed along $\langle 111\rangle$ and $\langle 110\rangle$ zone axis (d and e). In (a) and (d), the Fe and Cr containing regions are between the dashed white lines. 
interface (Figure $5 \mathrm{~d}, \mathrm{e}$ ). Here, $\mathrm{Cr}$ is clearly visible at interstitial sites in multiple $\mathrm{AO}$ and $\mathrm{BO}_{2}$ atomic planes near the interface, as indicated by the arrows in the high-resolution image in Figure 5e. Interestingly, $\mathrm{Fe}$ atoms diffuse away from the original interfacial $\mathrm{Fe}_{0.65} \mathrm{Ti}_{0.35} \mathrm{O}_{1.35}$ layer and into the film during STO overgrowth and are redistributed within several atomic planes. Thus, a strong Fe $L_{2,3}$ EELS feature is measured in regions of the film such as that shown by the circle in Figure 5a. Moreover, the $\mathrm{Fe}$ remains in the +2 charge state, as indicated by the $L_{3}: L_{2}$ branching ratio (Figure $5 \mathrm{c}$ ). However, the absence of intensity at interstitial sites in the ADF image (Figure 5b) reveals that $\mathrm{Fe}$ must be at lattice sites. Our instrumental resolution is not sufficiently high to determine which site (A or B) the Fe occupies.

In contrast to what was observed in the $\mathrm{Ti} 2 \mathrm{p}$ spectra for $\mathrm{Cr} /$ STO $(001),{ }^{7}$ there is no evidence for either charge transfer from $\mathrm{Fe}$ to $\mathrm{Ti}^{4+}$ to result in $\mathrm{Fe}^{3+}$ and $\mathrm{Ti}^{(3+\delta)+}$, or metallization of the surface when sub-ML $\mathrm{Fe}$ is deposited. Thus, an entirely different mechanism of interface formation appears to be operative with $\mathrm{Fe}$. The $\mathrm{Fe}_{x} \mathrm{Ti}_{1-x} \mathrm{O}_{2-x}$ layer that forms during nucleation of the first $\mathrm{ML}$ acts as a template for the subsequent overgrowth of epitaxial $\mathrm{Fe}$, despite the presence of a disordered layer, as seen in Figure 1d. This in turn results in slightly upward band bending of the STO $(\sim 0.2 \mathrm{eV})$, giving rise to the initiation of a Schottky barrier that dominates the electronic properties of the interface for thicker films.

To investigate the cause of the stark differences between the properties of $\mathrm{Fe}$ and $\mathrm{Cr}$ in STO, we calculated the relative stabilities and interaction energies for substitutional $\mathrm{Cr}$ and $\mathrm{Fe}$ occupying the B-type lattice sites $\left(\mathrm{Cr}_{\mathrm{Ti}}\right.$ and $\left.\mathrm{Fe}_{\mathrm{Ti}}\right)$, interstitial $\mathrm{Cr}$, $\mathrm{Fe}$, and $\mathrm{Ti}$ defects $\left(\mathrm{Cr}_{\mathrm{i}}, \mathrm{Fe}_{\mathrm{i}}, \mathrm{Ti}_{\mathrm{i}}\right)$, and oxygen vacancies $\left(V_{\mathrm{O}}\right)$. The results of these calculations and the resulting model of defect interconversion are summarized in Figure 6.

We assume that as an STO film is grown on top of the STO(001) exposed to sub-ML quantities of $\mathrm{Cr}$ or $\mathrm{Fe}$, some of these $\mathrm{Fe}$ and $\mathrm{Cr}$ atoms are located at the growth front and will occupy B sites. In order to preserve lattice neutrality, these $\mathrm{Fe}$ and $\mathrm{Cr}$ species must adopt a +4 oxidation state, which corresponds to the appearance of an acceptor electronic state

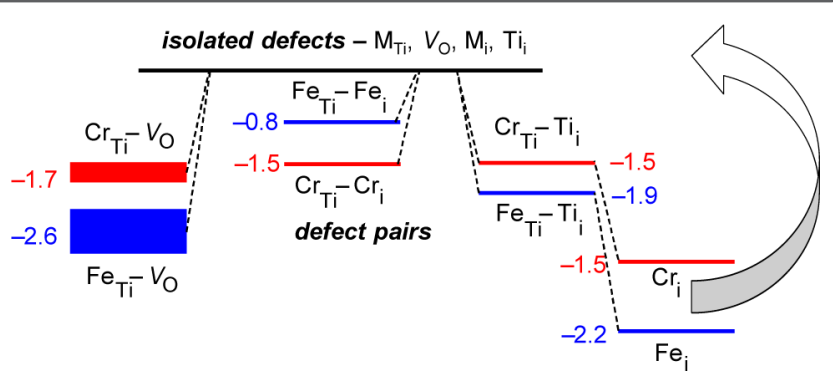

Figure 6. Association energies (in $\mathrm{eV}$ ) for $\mathrm{Cr}, \mathrm{Fe}, \mathrm{Ti}$, and $\mathrm{O}$ defect pairs in $\mathrm{SrTiO}_{3}$ and mechanisms of defect interconversion. Substitutional $\mathrm{M}^{4+}\left(\mathrm{M}_{\mathrm{T}}\right.$, where $\left.\mathrm{M}=\mathrm{Cr}, \mathrm{Fe}\right)$ binds to and is stabilized by oxygen vacancies $\left(V_{\mathrm{O}}\right)$, leading to $\mathrm{M}_{\mathrm{Ti}}-V_{\mathrm{O}}$ defect pairs (left side) that are more stable than $\mathrm{M}_{\mathrm{Ti}}-\mathrm{M}_{\mathrm{i}}$ pairs (middle). The stability of these defect pairs and the distribution of electrons associated with the vacancy depend on the distance between $\mathrm{M}_{\mathrm{Ti}}$ and $V_{\mathrm{O}}$. Right side: Interstitial $\mathrm{Ti}\left(\mathrm{Ti}_{\mathrm{i}}\right)$ interacts with substitutional $\mathrm{Cr}_{\mathrm{Ti}}$ and $\mathrm{Fe}_{\mathrm{Ti}}$ and displaces these cations to interstitial sites. Isolated interstitial $\mathrm{Cr}$ and $\mathrm{Fe}$ then diffuse to the growth front and re-enter the lattice as either $\mathrm{M}_{\mathrm{Ti}}-V_{\mathrm{O}}$ or $\mathrm{M}_{\mathrm{Ti}}-\mathrm{M}_{\mathrm{i}}$. On the basis of the calculated energies, $\mathrm{Fe}$ is more likely to occupy substitutional sties, whereas $\mathrm{Cr}$ is predicted to occupy both substitutional and interstitial sites. associated with these species and hybridized with the $\mathrm{O} 2 \mathrm{p}$ valence band. At high concentrations these acceptor states are known to be compensated by donor defects, such as $V_{\mathrm{O}}$, which is evidenced by significant deviations from the formal oxygen stoichiometry observed for $\mathrm{SrFeO}_{3}$ and $\mathrm{SrCrO}_{3}$. At relatively low concentrations, as is the case here, other types of donor defects can contribute to hole compensation associated with $\mathrm{Fe}(\mathrm{IV})$ and $\mathrm{Cr}(\mathrm{IV})$.

According to our calculations, the association energy of $V_{\mathrm{O}}$ with $\mathrm{Cr}_{\mathrm{Ti}}$ varies between $1.85 \mathrm{eV}$ for $\mathrm{Cr}_{\mathrm{Ti}}$ and $V_{\mathrm{O}}$ at a nearestneighbor site and $1.55 \mathrm{eV}$ for $\mathrm{Cr}_{\mathrm{Ti}}$ and $V_{\mathrm{O}}$ at a next-nearest neighbor site, leading to an average value of $1.7 \mathrm{eV}$. Similarly for $\mathrm{Fe}$, the $\mathrm{Fe}_{\mathrm{Ti}}-V_{\mathrm{O}}$ binding energy varies between 2.9 and 2.3 $\mathrm{eV}$, averaging to $2.6 \mathrm{eV}$. These are shown on the left side of Figure 6. Analysis of the charge-density distributions for these two types of configurations reveals that in the case of the $\mathrm{Cr}_{\mathrm{Ti}}-$ $V_{\mathrm{O}}$ pair, one of the electrons associated with the vacancy is transferred to the $\mathrm{Cr}_{\mathrm{Ti}}$, thus making it $\mathrm{Cr}$ (III), whereas another electron is predominantly associated with the vacancy site. In contrast, in the $\mathrm{Fe}_{\mathrm{Ti}}-V_{\mathrm{O}}$ pair, both $V_{\mathrm{O}}$ electrons are transferred to the $\mathrm{Fe}_{\mathrm{Ti}}$ site, making it $\mathrm{Fe}(\mathrm{II})$.

$\mathrm{Fe}_{\mathrm{Ti}}$ and $\mathrm{Cr}_{\mathrm{Ti}}$ can also be reduced by interstitial metal atoms, including $\mathrm{Ti}, \mathrm{Fe}$, and $\mathrm{Cr}$. Our calculations reveal that $\mathrm{Fe}_{\mathrm{Ti}}-\mathrm{Fe}_{\mathrm{i}}$ and $\mathrm{Cr}_{\mathrm{Ti}}-\mathrm{Cr}_{\mathrm{i}}$ donor pair formation result in energy gains of 0.8 and $1.5 \mathrm{eV}$, respectively (middle section of Figure 6). Likewise, both $\mathrm{Fe}_{\mathrm{Ti}}$ and $\mathrm{Cr}_{\mathrm{Ti}}$ form stable donor pairs with $\mathrm{Ti}_{\mathrm{i}}$, exhibiting binding energies of 1.9 and $1.5 \mathrm{eV}$, respectively (right side of Figure 6). However, $\mathrm{Ti}_{\mathrm{i}}$ can displace $\mathrm{Fe}_{\mathrm{Ti}}$ and $\mathrm{Cr}_{\mathrm{Ti}}$ from the $\mathrm{B}$ sites they occupy to interstitial positions, and then occupy the now vacant $B$ site, with additional gains of 2.2 and $1.5 \mathrm{eV}$, respectively (right side of Figure 6). Once this process occurs, interstitial $\mathrm{Cr}_{\mathrm{i}}$ and $\mathrm{Fe}_{\mathrm{i}}$ become available for additional interactions at the growth front.

From these calculations, it is clear that $\mathrm{Fe}_{\mathrm{Ti}}-V_{\mathrm{O}}$ is more stable than $\mathrm{Fe}_{\mathrm{Ti}}-\mathrm{Fe}_{\mathrm{i}}$ and should dominate. In contrast, $\mathrm{Cr}_{\mathrm{Ti}}-V$ $\mathrm{O}$ and $\mathrm{Cr}_{\mathrm{Ti}}-\mathrm{Cr}_{\mathrm{i}}$ have comparable binding energies and should both be present. Bader analysis indicates that $\mathrm{Cr}_{\mathrm{i}}$ should be +2 . Finally, we note that the binding of $\mathrm{Cr}_{\mathrm{Ti}}-\mathrm{Ti}_{\mathrm{i}}$ pairs is transient; it results in the formation of isolated interstitial species, which are also transient.

\section{SUMMARY}

We have investigated epitaxial $\mathrm{Fe} / n-\mathrm{SrTiO}_{3}(001)$ heterojunctions and have compared the results with analogous data for epitaxial $\mathrm{Cr} / n-\mathrm{SrTiO}_{3}(001)$ prepared under the same experimental conditions. Both $\mathrm{Cr}$ and $\mathrm{Fe}$ are bcc metals and have nearly identical lattice parameters. Both grow epitaxially on STO(001) and neither interface is atomically abrupt; some metal diffuses into the STO in both cases. However, the electronic properties of the two interfaces are completely different, despite the metals having very similar work functions. Fe forms a $0.5 \mathrm{eV}$ Schottky barrier, whereas $\mathrm{Cr}$ forms a lowresistance ohmic contact. This stark contrast can be traced to differences in interface chemistry. $\mathrm{Fe}$ atoms displaces $\mathrm{Ti}$ and occupy B sites as $\mathrm{Fe}(\mathrm{II})$, a result confirmed by both experiment and first-principles modeling, and cause partial disordering of the interfacial region when thicker films are grown. In contrast, $\mathrm{Cr}$ atoms appear to occupy interstitial sites, transferring charge to B-site $\mathrm{Ti}(\mathrm{IV})$ ions in the process, and metallizing the surface. First-principles calculations suggest that there may also be some $\mathrm{Cr}$ at B sites in the STO lattice near the interface. However, such species are as yet undetected experimentally. Bader analysis yields valences of +3 for $\mathrm{Cr}_{\mathrm{Ti}}$ and +2 for $\mathrm{Cr}_{\mathrm{i}}$. This 
comparative study illustrates how critically important understanding interface chemistry and structure are in determining the electronic properties of a metal/wide-gap oxide heterojunction.

\section{AUTHOR INFORMATION}

\section{Corresponding Author}

*E-mail: sa.chambers@pnnl.gov.

\section{Author Contributions}

S.A.C. and Y.D. carried out the epitaxial film growth and in situ XPS measurements, M.G. did the STEM/EELS, T.C.D. carried out the transport measurements, and S.P.H. and P.V.S. performed the $\mathrm{ab}$ initio calculations. All authors contributed to and approved the final version of the manuscript.

\section{Notes}

The authors declare no competing financial interest.

\section{ACKNOWLEDGMENTS}

This work was supported by the U.S. Department of Energy, Office of Basic Energy Sciences, Division of Materials Sciences and Engineering under Award \#10122. The work described was conducted in the William R. Wiley Environmental Molecular Sciences Laboratory (EMSL), a national scientific user facility sponsored by DOE's Office of Biological and Environmental Research and located at PNNL. PNNL is operated by Battelle for the DOE under Contract DE-AC05-76RLO1830. S.P.H. was supported by the EPSRC Grant No.EP/I009973/1. Access to the HECToR high-performance computing facility was made available via S.P.H. membership of the U.K.'s HPC Materials Chemistry Consortium, which was funded by EPSRC (EP/ F067496).

\section{REFERENCES}

(1) Robertson, J.; Chen, C. W. Schottky barrier heights of tantalum oxide, barium strontium titanate, lead titanate and strontium bismuth tantalate. Appl. Phys. Lett. 1999, 74, 1168.

(2) Mrovec, M.; Albina, J. M.; Meyer, B.; Elsasser, C. Schottky barriers at transition-metal $/ \mathrm{SrTiO}_{3}(001)$ interfaces. Phys. Rev. B 2009, $79,245121$.

(3) Stocker, H.; Zschornak, M.; Seibt, J.; Hanzig, F.; Wintz, S.; Abendroth, B.; Kortus, J.; Meyer, D. C. Formation of Schottky-type metal/SrTiO(3) junctions and their resistive properties. Appl. Phys. A: Mater. Sci. Process. 2010, 100, 437.

(4) Spinelli, A.; Torija, M. A.; Liu, C.; Jan, C.; Leighton, C. Electronic transport in doped $\mathrm{SrTiO}(3)$ : Conduction mechanisms and potential applications. Phys. Rev. B 2010, 81, 155110.

(5) Son, J.; Moetakef, P.; Jalan, B.; Bierwagen, O.; Wright, N. J.; Engel-Herbert, R.; Stemmer, S. Epitaxial $\mathrm{SrTiO}_{3}$ films with electron mobilities exceeding 30,000 $\mathrm{cm}^{2} \mathrm{~V}^{-1} \mathrm{~s}^{-1}$. Nat. Mater. 2010, 9, 482.

(6) Capan, C.; Sun, G. Y.; Bowden, M. E.; Chambers, S. A. Epitaxial $\mathrm{Cr}$ on $\mathrm{n}-\mathrm{SrTiO}_{3}(001)$-An ideal Ohmic contact. Appl. Phys. Lett. 2012, $100,052106$.

(7) Chambers, S. A.; Gu, M.; Sushko, P. V.; Yang, H.; Wang, C. M.; Browning, N. D. Ultralow Contact Resistance at an Epitaxial Metal/ Oxide Heterojunction Through Interstitial Site Doping. Adv. Mater. 2013, 25, 4001.

(8) Perdew, J. P.; Burke, K.; Ernzerhof, M. Generalized gradient approximation made simple. Phys. Rev. Lett. 1996, 77, 3865.

(9) Silly, F.; Castell, M. R. Fe nanocrystal growth on $\mathrm{SrTiO}_{3}(001)$. Appl. Phys. Lett. 2005, 87, 063106.

(10) Kamaratos, M.; Vlachos, D.; Foulias, S. D. Development and characterization of $\mathrm{Fe}$ ultrathin films on the $\mathrm{SrTiO}_{3}(100)$ surface. J. Phys.: Condens. Matter 2008, 20, 315009.

(11) CRC Handbook of Chemistry and Physics CRC Press: Boca Raton, FL, 2008.
(12) Kresse, G.; Furthmuller, J. Efficient iterative schemes for ab initio total-energy calculations using a plane-wave basis set. Phys. Rev. B 1996, 54, 11169.

(13) Kresse, G.; Joubert, D. From ultrasoft pseudopotentials to the projector augmented-wave method. Phys. Rev. B 1999, 59, 1758.

(14) Blochl, P. E. Projector Augmented-Wave Method. Phys. Rev. B 1994, 50, 17953.

(15) Perdew, J. P.; Ruzsinszky, A.; Csonka, G. I.; Vydrov, O. A.; Scuseria, G. E.; Constantin, L. A.; Zhou, X. L.; Burke, K. Restoring the density-gradient expansion for exchange in solids and surfaces. Phys. Rev. Lett. 2008, 100, 136406.

(16) Bader, R. F. W. Atoms in Molecules - A Quantum Theory; Oxford University Press: New York, 1990.

(17) Nabi, H. S.; Pentcheva, R. Energetic stability and magnetic coupling in $\left(\mathrm{Cr}_{1-x} \mathrm{Fe}_{x}\right)_{2} \mathrm{O}^{-3}$ : Evidence for a ferrimagnetic ilmenite-type superlattice from first principles. Phys. Rev. B 2011, 83, 214424.

(18) Cosandey, F.; Su, D.; Sina, M.; Pereira, N.; Amatucci, G. G. Fe valence determination and $\mathrm{Li}$ elemental distribution in lithiated FeO0.7F1.3/C nanocomposite battery materials by electron energy loss spectroscopy (EELS). Micron 2012, 43, 22. 